\title{
Redução, refinamento e substituição do uso de animais em estudos toxicológicos: uma abordagem atual
}

\author{
Karen Cristine Ceroni Cazarin ${ }^{1,3 *}$, Cristiana Leslie Corrêa ${ }^{1,2}$, Flávio Ailton Duque Zambrone ${ }^{1,3}$ \\ ${ }^{1}$ PLANITOX - Planejamento, Assessoria e Informação em Toxicologia, ${ }^{2}$ Faculdade de Ciências Farmacêuticas, \\ Centro de Ciências da Vida, Pontifícia Universidade Católica de Campinas, ${ }^{3}$ Departamento de Medicina Preventiva \\ e Social, Faculdade de Ciências Médicas, Universidade Estadual de Campinas
}

*Correspondência:

K. C. C. Cazarin

Faculdade de Ciências Médicas -

UNICAMP

Depto. de Medicina Preventiva

Rua José Inicêncio de Campos, 153, 3o

andar, Cambuí

13024-230 - Campinas - SP, Brasil

E-mail: karen.cazarin@planitox.com.br.
A avaliação da toxicidade de uma substância é realizada com o objetivo de predizer os efeitos nocivos que a mesma poderá desencadear quando da exposição humana pelas diversas vias. Para cumprir este propósito, o modelo animal é o mais utilizado nos estudos toxicológicos e requerido nos processos investigativos. Entretanto, a utilização de animais na pesquisa tem sido razão de diversas discussões em função do grande número necessário e do sofrimento causado, principalmente em relação aos estudos de toxicidade aguda. Existe uma tendência mundial para reavaliar a utilização de animais nos experimentos, concretizada a partir de um programa denominado de $3 R s$ (Reduction, Refinement, Replacement), que objetiva além de diminuir o número de animais, minimizar a dor e o desconforto e buscar alternativas para a substituição dos testes in vivo. Diversas metodologias alternativas já foram implantadas, sendo este um processo complexo que abrange desde o seu desenvolvimento até sua aceitação regulatória e adoção por diversas organizações. Sendo assim, o presente trabalho apresenta abordagem atualizada do Programa 3Rs, com ênfase na sua evolução histórica e nos processos de implantação e validação de métodos alternativos, principalmente aplicados no contexto da avaliação da toxicidade, enfatizando sua importância e utilidade frente à tendência global de harmonização.
Unitermos:

- Programa 3Rs

- Animais de experimentação

- Toxicologia - estudos

\section{INTRODUÇÃO}

Os estudos toxicológicos apresentam como principal objetivo a predição dos possíveis efeitos adversos, que podem se manifestar quando da exposição humana à determinada substância química, seja ela um medicamento, um praguicida, um agente químico industrial ou outros
(Koeter, 1993; Stokes, 2002; Meyer, 2003). Por esta razão, tais estudos são sempre requeridos nos processos investigativos, desde o desenvolvimento de produtos até seu registro e comercialização, sendo os modelos animais os mais utilizados para este propósito (Ecobichon, 1997; Stokes, 2002; Meyer, 2003).

Em geral, o uso de animais em procedimentos cien- 
tíficos é voltado para o diagnóstico $(<5 \%)$, para a educação, principalmente nas Universidades, incluindo a dissecação animal (25\%), para o desenvolvimento e para os testes de toxicidade de medicamentos e de produtos biológicos (30 a 40\%) e de novos produtos (10\%) (Humane..., 2003). Investigação realizada pela Research Defence Society (RDS) demonstrou que no ano de 2002 mais de 2 $1 / 2$ milhões de animais foram utilizados em procedimentos científicos na Grã Bretanha, sendo os roedores as espécies de maior relevância (Tabela I) (Matfield, 2003). Vale ressaltar que a estimativa do número de animais utilizados em pesquisas anualmente é altamente especulativa. A última estimativa oficial dos Estados Unidos, realizada há mais de 15 anos, revelou a utilização de cerca de 17 a 22 milhões de animais (Stephens et al., 2001).

TABELA I - Porcentagem de animais utilizados na GrãBretanha, em procedimentos científicos, no ano de 2002

\begin{tabular}{lc}
\hline Espécies & $\mathbf{\%}$ \\
\hline Ratos e camundongos & 84 \\
Peixes, anfíbios, répteis e pássaros & 12 \\
Ovelhas, vacas, porcos e outros animais & 2,3 \\
$\quad$ de grande porte & \\
$\begin{array}{l}\text { Pequenos mamíferos e outros roedores } \\
\quad \text { coelho e furão) }\end{array}$ & 1,5 \\
Cães e gatos (espécies de laboratório) & 0,3 \\
Macacos & 0,15 \\
\hline
\end{tabular}

Fonte: Research Defence Society (RDS)

Entretanto, o uso disseminado de animais na pesquisa tem sido motivo de diversas discussões, principalmente de caráter ético, em função do grande número de animais requerido e do sofrimento causado durante alguns tipos de experimento (White, 2001; Meyer, 2003). Por esta razão, a reavaliação da utilização de animais nos experimentos é tendência mundial, concretizada a partir da fundação de diversas Instituições, que objetivam desenvolver e validar novos métodos, e da implementação regulatória de testes alternativos em diversos países, a fim de legalizar e harmonizar o uso dos mesmos (Schechtman, 2002; Russel; Burch, 1992; Balls, 1994).

Todas essas ações tiveram início a partir do surgimento de um programa internacionalmente reconhecido denominado de $3 R s$ (Reduction, Refinement, Replacement), que objetiva, além de diminuir o número de animais utilizados na pesquisa, minimizar a dor e o desconforto e buscar alternativas para a substituição dos testes in vivo (Schechtman, 2002; Russel; Burch, 1992; Balls, 1994; Flecknell,1994).
Sendo assim, o propósito do presente artigo foi realizar abordagem atualizada sobre o Programa 3Rs, com ênfase na sua evolução histórica e nos processos de implantação e validação de métodos alternativos, principalmente aplicados no contexto da avaliação da toxicidade.

\section{PROGRAMA 3Rs: CONCEITOS E OBJETIVOS}

O programa $3 R s$ é assim denominado em função das iniciais, em inglês, de seus principais objetivos: 1 ) redução (Reduction), 2) refinamento (Refinement) e 3) substituição (Replacement), que de forma resumida significam a redução do número de animais utilizados na pesquisa, a melhora na condução dos estudos, no sentido de reduzir o sofrimento ao mínimo possível, e a busca de métodos alternativos que, por fim, substituam os testes in vivo. Os dois primeiros representam os objetivos a curto-prazo e o último, a meta máxima a ser alcançada (Dipasquale; Hayes, 2001; Three..., 2000).

No sentido de concretizar tais objetivos, diversas ações e metas são propostas, algumas das quais descritas na tabela II, e que muito têm contribuído para a aceitação e a evolução desta filosofia na área experimental.

Vale ressaltar que métodos alternativos in vitro validados, genuínos e aceitos com propósito regulatório para repor testes conduzidos com animais, ainda são considerados muito mais uma meta do que realidade, embora tais conceitos já sejam amplamente incorporados por pesquisadores, por organizações não governamentais e por algumas agências regulamentadoras.

Desta forma, o propósito principal do programa 3Rs é servir como um conceito unificador, um desafio e uma oportunidade para a obtenção de benefícios científicos, econômicos e humanitários (Balls, 2000).

\section{EVOLUÇÃO HISTÓRICA}

O desenvolvimento e a utilização de metodologias alternativas aos estudos realizados com animais de laboratório é meta de longa data dentro da comunidade científica. Data de 1876, na Grã-Bretanha, a primeira legislação criada especificamente para regulamentar a utilização de animais em experimentos (Stephens et al., 2001).

Porém, o marco inicial do programa de redução, refinamento e substituição (3Rs) foi em 1954, num projeto iniciado pela Federação das Universidades para o Bemestar Animal (The Universities Federation for Animal Welfare - UFAW's), que resultou na publicação dos Princípios das Técnicas Experimentais Humanas (The principles of Humane Experimental Technique) em 1959, por Willian Russell e Rex Burch, considerados os 
TABELA II -Ações e metas fundamentais para o cumprimento dos objetivos do Programa 3 Rs

\begin{tabular}{|c|c|c|}
\hline REDUÇÃO & REFINAMENTO & SUBSTITUIÇÃO \\
\hline $\begin{array}{l}\text { - desenvolver novos protocolos } \\
\text { com a utilização de menor número } \\
\text { de animais por experimento; } \\
\text { - evitar a replicação dos estudos } \\
\text { conduzidos in vivo; } \\
\text { - evitar a condução de estudos } \\
\text { utilizando modelos animais que } \\
\text { têm demonstrado irrelevância na } \\
\text { extrapolação dos efeitos para a } \\
\text { espécie humana; } \\
\text { - desenvolver metodologias ex vivo } \\
\text { e in vitro, com o intuito de utilizá-las } \\
\text { como triagem (screening) para a } \\
\text { identificação do efeito de relevância } \\
\text { e para posterior investigação; } \\
\text { - aperfeiçoar a qualidade técnica } \\
\text { dos ensaios; } \\
\text { • obter o maior número possível de } \\
\text { informações relevantes em um } \\
\text { pequeno número de animais. }\end{array}$ & $\begin{array}{l}\text { •utilizar novas metodologias a } \\
\text { partir do avanço técnico e científico } \\
\text { e da investigação direcionada para os } \\
\text { "endpoints" humanos (efeitos de } \\
\text { maior relevância). Por esta razão, } \\
\text { elementos qualitativos devem ser } \\
\text { empregados, além da preocupação } \\
\text { com a qualidade de vida do animal } \\
\text { durante os procedimentos e a } \\
\text { necessidade de reduzir o risco e o grau } \\
\text { de sofrimento dos mesmos. }\end{array}$ & $\begin{array}{l}\text { - desenvolver métodos alternativos à } \\
\text { experimentação animal, tais como } \\
\text { ensaios in vitro, inclusive com } \\
\text { utilização de células humanas. }\end{array}$ \\
\hline
\end{tabular}

Fonte: Balls, 1994; Flecknell, 1994; Festing, 1994; Three..., 2000; Schlede et al. [entre 1998 e 2003].

iniciadores desta filosofia (Russel; Burch, 1992; Executive..., 2000; Stephens et al., 2001; Zurlo, 2002; Spielmann, 2002).

Pouca atenção foi dispensada à concepção dos $3 R s$ na década de 60. Alguns acontecimentos nesta área ocorreram nos anos 70 , porém, somente a partir de 1980 foi observada a introdução de leis e convenções nacionais e internacionais baseadas no programa 3Rs. No final da década de 1980, novas leis e protocolos foram criados e adotados em diversos países, não somente reconhecendo a concepção de Russell e Burch, mas também identificando as obrigações legais e morais envolvidas na busca de reduzir, refinar e substituir, sempre que possível, os procedimentos envolvendo os animais de experimentação (Executive..., 2000).

Todo este movimento em prol do programa 3Rs resultou na fundação de diversas instituições, no oferecimento de encontros/seminários e no crescente estímulo de publicações científicas, a fim de promover o desenvolvimento, a validação e a discussão de alternativas para a substituição das técnicas usuais.
Os principais acontecimentos históricos envolvendo o programa 3Rs e a introdução de métodos alternativos podem ser observados na Tabela III (Russel; Burch, 1992; Executive..., 2000; Stephens et al., 2001; Zurlo, 2002; Alternative, [entre 1997 e 2003]).

\section{ESTUDOS TOXICOLÓGICOS FRENTE AO PROGRAMA 3Rs}

A avaliação da toxicidade visa caracterizar os efeitos adversos inerentes a determinado agente químico (Corrêa et al., 2003). Sendo assim, os testes toxicológicos normalmente requeridos com propósito regulatório incluem: toxicidade aguda, toxicidade sub-crônica, toxicidade crônica, mutagênese, carcinogênese, reprodução e teratogênese, toxicocinética, efeitos locais sobre a pele e olhos, sensibilização cutânea e ecotoxicidade (Barros, Davino, 2003; Spielmann, 2002).

Um dos ensaios toxicológicos mais duramente criticado e discutido frente ao programa 3Rs, em virtude do grande número de animais requerido e do sofrimento cau- 
TABELA III - Descrição de alguns dos principais acontecimentos históricos relacionados ao programa 3Rs e à introdução de métodos alternativos

\begin{tabular}{ll}
\hline DATA & ACONTECIMENTO \\
\hline $\mathbf{1 9 5 4}$ & $\begin{array}{l}\text { A Federação das Universidades para o Bem-estar Animal (The Universities Federation for Animal Welfare-UFAW) } \\
\text { constituiu um comitê para estudar as técnicas humanas utilizadas nos experimentos com animais de laboratório. }\end{array}$ \\
$\mathbf{1 9 5 9}$ & $\begin{array}{l}\text { O estudo de Russell e Burch é publicado, contendo uma discussão detalhada do proposto programa 3Rs. } \\
\mathbf{1 9 6 3} \\
\text { A primeira edição do The Guide for the Care and the Use of Laboratory Animals, escrito pela Academia Nacional de } \\
\text { Ciências, é publicada pelo Instituto Nacional de Saúde. }\end{array}$ \\
& $\begin{array}{l}\text { O fundo de substituição de animais em experimentos médicos (The Fund of the Replacement of Animals in Medical } \\
\text { alternativinos. FRAME) é formado no Reino Unido para promover, junto à comunidade científica, a idéia de métodos }\end{array}$
\end{tabular}

O FRAME publicou um documento esboçando algumas metodologias de substituição, tais como modelos computadorizados, estudos em culturas de células e uso de organismos inferiores.

1971 Bruce Ames, da Universidade da Califórnia em Berkeley, introduziu um teste in vitro para mutagênese usando a Salmonella typhimurium.

1975 A Academia Nacional de Ciências dos EUA (The U.S. National Academy of Sciences) presidiu o maior encontro científico sobre os testes alternativos nos Estados Unidos.

1979 Foi oficializado o primeiro fundo governamental para os testes alternativos: o governo da Suécia distribuiu $\$ 90.000$ para investimento.

1980 Surge primeira campanha contra os testes de irritação ocular conduzidos em coelhos.

1983 O FDA dos Estados Unidos (The Food and Drug Administration) anunciou formalmente que não iria mais requerer o teste clássico de toxicidade aguda (DL50).

1986 Nova legislação sobre animais (procedimentos científicos) do Reino Unido (UK's Animals Act) substituiu a lei do ano de 1876. Conselho de Ministros da Comunidade Européia legaliza a diretiva EC 86/609, requerendo que os países membros desenvolvam legislação que promova os 3Rs. A OECD* anunciou mudanças nos protocolos de toxicidade aguda oral e dérmica e iniciou discussão sobre os métodos alternativos.

1991 A OECD adotou o teste de doses fixadas (Fixed Dose Method) como uma alternativa ao teste de DL50 clássico;Foi fundado o Centro Europeu de Validação de Métodos Alternativos (European Center for the Validation of Alternative Methods-ECVAM).

$1993 \quad 1^{\circ}$ Congresso Mundial sobre os Testes Alternativos e o Uso de Animais nas Ciências da Vida: Educação, Pesquisa e Testes (The first World Congress on Alternatives and Animal Use in the Life Sciences: Education, Research and Testing), em Baltimore, EUA.

$199622^{\circ}$ Congresso Mundial sobre os Testes Alternativos e o Uso de Animais nas Ciências da Vida: Educação, Pesquisa e Testes (The first World Congress on Alternatives and Animal Use in the Life Sciences: Education, Research and Testing), em Utrecht, Holanda.

1997 O governo federal dos EUA funda o Comitê de Coordenação Interagências para Validação de Métodos Alternativos - ICCVAM (Interagency Coordinating Committee on the Validation of Alternative Methods), com participação do NIEHS* e EPA*.

1998

O ECVAM aprova os seguintes métodos alternativos: teste 3T3 NRU PT - como alternativa na avaliação de fototoxicidade. Episkin e o TER (transepithelial electrical resistance) - na avaliação de corrosão dérmica. O Centro Interagências do Programa Nacional de Toxicologia para Avaliação de Métodos Toxicológicos Alternativos (NICEATM) foi fundado para dar suporte ao ICCVAM.

1999

$3^{\circ}$ Congresso Mundial sobre os Testes Alternativos e o Uso de Animais nas Ciências da Vida: Educação, Pesquisa e Testes (The first World Congress on Alternatives and Animal Use in the Life Sciences: Education, Research and Testing), em Bologna, Itália.

2000 A OECD anuncia oficialmente os planos de extinguir o teste de DL50 (Test Guideline 401) de seus protocolos, em favor dos três métodos alternativos existentes.

Cancelamento do Guideline 401 pela OECD.

*OECD - Organization for Economic Co-operation and Development; NIEHS - National Institute of Environmental Health Sciences; EPA Environmental Protection Agency dos Estados Unidos 
sado, é o ensaio de toxicidade aguda oral (guideline OECD 401), o qual foi primeiramente adotado em 1981, com o objetivo de determinar a dose única, derivada de cálculos estatísticos, que pode causar a morte de $50 \%$ de uma dada população, em condições experimentais definidas $\left(\mathrm{DL}_{50}\right)$ (Botham, 2002). Em 1986, a Organization for Economic Co-operation and Development (OECD) anunciou alterações nos guidelines de toxicidade aguda oral e dérmica, iniciando as discussões a respeito dos testes alternativos (Humane..., 2003).

Em 1983, o Food and Drug Administration dos Estados Unidos (FDA) já havia anunciado que não mais requereria o teste clássico de toxicidade aguda $\left(\mathrm{DL}_{50}\right)$ nos processos regulatórios e, em 1986, emitiu um relatório demonstrando redução de $96 \%$ do uso do método clássico de toxicidade aguda $\left(\mathrm{DL}_{50}\right)$ referente ao ano de 1985 , quando comparado ao período de 1975 a 1979 (Humane..., 2003).

Os primeiros guidelines de métodos alternativos validados e adotados pela OECD foram relacionados com a determinação da toxicidade aguda oral, sendo eles: o teste de doses fixadas (Fixed Dose Method - OECD 420), adotado em 1992, o método de classe tóxica aguda (Acute Toxic Class Method - OECD 423), adotado em 1996, e o teste Up-and-down (Up-and-down procedure - OECD 425), adotado em 1998 (Botham, 2002; Organization..., 1987, 1992, 1996, 2000, 2002a, b, c). Estes testes têm demonstrado que informações adequadas de toxicidade aguda e letalidade podem ser obtidas utilizando número menor de animais do que o teste clássico de $\mathrm{DL}_{50}$. Embora o endpoint principal ainda seja a letalidade, estes testes cumprem a meta de reduzir o número e o sofrimento dos animais e em alguns casos fornecem informação adequada para a classificação toxicológica das substâncias estudadas (Dipasquale; Hayes, 2001). Em 2001, estes protocolos foram atualizados, sendo estabelecido período de 1 ano para a interrupção de uso (phaseout) do guideline 401 referente à toxicidade aguda oral, o qual foi cancelado pela OECD em dezembro de 2002 (Botham, 2002). No Reino Unido, o ensaio de toxicidade aguda oral (OECD 401) foi banido em outubro de 1999 (Matfield, 2003; Koeter, [2003?])

Da mesma forma, a avaliação do potencial irritante dérmico e ocular, no contexto dos métodos alternativos, passou a ser discutida, sendo posteriormente sugerida a realização de testes preliminares e a condução de testes seqüenciais, ou seja, dos testes mais simplificados para os mais sofisticados (Stitzel, 2002). Anteriormente à condução dos estudos em animais de experimentação, as propriedades físico-químicas e os dados de ensaios in vitro validados devem ser considerados (Koeter, [2003?];
Olden..., 2004). No caso dos estudos de irritabilidade/ corrosividade aguda dérmica, alguns métodos in vitro, aceitos por autoridades regulamentadoras, incluem: teste de resistência elétrica transcutânea (Transcutaneous Electrical Resistance Test (TER) - OECD- 430); teste com modelo de epiderme humana (Human skin model test-EpiDerm $^{T M}$ - OECD-431); teste de fototoxicidade in vitro em células $3 \mathrm{~T} 3 \mathrm{NRU}$ (In vitro $3 T 3 \mathrm{NRU}$ phototoxicity test - OECD- 432); CORROSITEX ${ }^{T M}$ (ICCVAM); Episkin ${ }^{T M}$ (ICCVAM) e teste de integridade dérmica (Skin integrity function test - SIFT) (Stokes, 2002; Stitzel, 2002). Dentre os métodos alternativos para avaliar a irritabilidade/corrosividade aguda ocular, o teste em membrana corioalantóica de ovos de galinha (HETCAM: Hen Egg Test - Chorioallantoic Membrane Test) é um dos mais estudados.

Considera-se que, não apenas para a determinação do potencial de irritação, mas para a avaliação geral da toxicidade de uma determinada substância, a avaliação por etapas (tier approach) representa ferramenta de grande utilidade para se alcançar os objetivos do programa 3Rs, além de aperfeiçoar o processo de identificação do perigo e de reduzir o tempo e os custos empregados nesta avaliação (Botham, 2001; Stitzel, 2002). Entretanto, devese ressaltar a necessidade de avaliadores qualificados, já que cabe a eles o julgamento da condução do teste consecutivo a cada etapa (Koeter, [2003?]).

Os novos métodos, assim como os revisados (updated), fornecem avaliação mais acurada a respeito dos efeitos potenciais de determinadas substâncias químicas sobre a saúde humana e sobre o meio ambiente. Isto inclui o desenvolvimento de métodos que identificam novos endpoints toxicológicos, a clarificação de mecanismos de toxicidade, o aperfeiçoamento da metodologia de ensaio (ex: redução do tempo e custo), além de atender o objetivo de redução, refinamento e substituição dos estudos conduzidos em animais (Interagency..., 2003).

\section{PROCESSOS DE VALIDAÇÃO E DE IMPLEMENTAÇÃO REGULATÓRIA DOS MÉTODOS ALTERNATIVOS}

A fim de contemplar os objetivos do programa 3Rs, diversas metodologias têm sido desenvolvidas e apresentadas internacionalmente. Entretanto, para que as mesmas sejam utilizadas é necessária a elaboração de protocolos de estudo (guidelines), os quais são publicados apenas quando a nova proposta de ensaio já fora avaliada, validada e aprovada pelos órgãos competentes. $\mathrm{O}$ segundo passo consiste no reconhecimento e na utilização de tal metodologia dentro da proposta regulatória de cada país. 
Diversos países, tais como os Estados Unidos e membros da comunidade européia (ex: Reino Unido, Alemanha e a maior parte dos países membros) já aprovaram a utilização de métodos alternativos no processo de registro de novas substâncias, assim como a substituição de alguns métodos clássicos por outros que se enquadrem na proposta dos 3Rs, como por exemplo, a substituição do estudo clássico de toxicidade aguda oral, a partir da determinação da $\mathrm{DL}_{50}$ (Organization..., 1987).

Portanto, para que um novo método seja aceito, este deve passar por avaliação a fim de estabelecer sua relevância para implementação e sua confiabilidade (Stokes, 2002). Para que isso seja possível, faz-se necessária a harmonização dos processos de validação, por intermédio de Comitês Internacionais (Schechtman, 2002).

Nos Estados Unidos, o Comitê Organizador InterAgências para Validação de Métodos Alternativos, o ICCVAM (Interagency Coordinating Committee on the Validation of Alternative Methods), foi estabelecido em 1997 pela Diretoria do Instituto Nacional de Ciências para a Saúde Ambiental (NIEHS). Esta decisão foi tomada com base na Lei Pública 103-43, que proveu o desenvolvimento e o estabelecimento de critérios para a validação e aceitação regulatória de novos ensaios toxicológicos, sob responsabilidade do NIEHS (Interagency..., 1997, 2001, 2003).

O ICCVAM é composto por 15 Agências regulatórias e de pesquisa, dentre as quais incluem-se a Environmental Protection Agency dos Estados Unidos (EPA), a Food and Drug Administration dos Estados Unidos (FDA) e o Agency for Toxic Substances and Disease Registry (ATSDR), sendo que estas fornecem ou utilizam informações dos testes toxicológicos para o processo de avaliação do risco. O Comitê coordena, através das Agências, a discussão relativa ao desenvolvimento, validação, aceitação e harmonização nacional e internacional dos ensaios toxicológicos, por intermédio do governo federal dos Estados Unidos (Interagency..., 1999, 2001, 2003).

Em 1998, o Centro Inter-Agências para a Avaliação de Métodos Toxicológicos Alternativos (NICEATM) foi estabelecido pelo National Toxicology Program (NTP), com o objetivo de fornecer suporte operacional ao ICCVAM, e para executar as atividades relacionadas ao Comitê, tais como peer views e workshops de métodos alternativos de interesse para as Agências Federais (Schechtman, 2002; Interagency..., 2001, 2003).

Assim, o ICCVAM e o NICEATM promovem a validação, a regulamentação e aceitação de métodos alternativos que melhor predizem os efeitos ecológicos e para a saúde humana, em comparação aos métodos usuais.
Portanto, os esforços de ambas as Agências são direcionados ao aperfeiçoamento da avaliação da toxicidade, à redução dos custos e do tempo despendidos na condução dos estudos, e à redução, ao refinamento e à substituição do uso de animais (Interagency..., 1997, 2001, 2003).

$\mathrm{Na}$ Europa, o órgão responsável pelo processo de validação é o European Centre for the Validation of Alternative Methods (ECVAM), que foi criado em outubro de 1991, pela Comissão para o Conselho e Parlamento (Communication from the Commission to the Council and the Parliament), em resposta à Diretiva Européia 86/ $609 / \mathrm{EEC}$, relativa à proteção dos animais utilizados na experimentação e em outros procedimentos científicos, onde foi solicitado o apoio da Comissão e dos Estados Membros, nos processos de desenvolvimento, validação e aceitação de novos métodos com o objetivo de reduzir, refinar e substituir o uso dos animais de laboratório (European ..., [200-?]).

Assim, os objetivos específicos do ECVAM são: 1) coordenar o processo de validação de métodos alternativos na União Européia, 2) proceder à troca de informações no desenvolvimento de métodos alternativos, 3) estabelecer, manter e administrar um banco de dados dos procedimentos alternativos, 4) promover o diálogo entre legisladores, indústrias, cientistas, consumidores e grupos voltados para a proteção animal (ECVAM..., [200-?]); Worth, Balls, 2002; Schechtman, 2002).

O trabalho do Centro Europeu é focado no desenvolvimento e na avaliação de métodos in vitro (exemplo: culturas de células e tecidos), no uso de modelos computadorizados com base na relação estrutura-atividade (REA) e nos modelos fisiológicos e cinéticos. Embora o ECVAM desempenhe papel ativo no programa de pesquisa, seu principal objetivo é a validação de métodos alternativos, direcionado às atividades pré-normativas. Isso é possível, através de seu Comitê Científico Consultivo (Scientific Advisory Committee/ESAC), que é composto por representantes de todos os Estados Membros (Áustria, Bélgica, Dinamarca, Finlândia, França, Alemanha, Grécia, Irlanda, Itália, Luxemburgo, Países Baixos, Portugal, Espanha, Suécia e Reino Unido), de associações das indústrias, da Academia, de associações para o bem-estar animal (Eurogroup), de departamentos da Comissão Européia e de espectadores de outras comissões de interesse (NICEATM e ICCVAM) (European..., [200-?]).

Depois de finalizada a fase de validação, o método proposto passa por uma avaliação independente, ou seja, por instituições que não estiveram envolvidas durante todo o processo de pesquisa, de desenvolvimento e da validação do método, para emissão de um parecer. Quando este 
é favorável, o método proposto é publicado e enviado para os órgãos competentes para implementação regulatória.

Em linhas gerais, o processo anteriormente descrito demonstra as etapas pelas quais a nova metodologia deve enfrentar antes de ser aceita, sendo que o período entre a etapa de pré-validação e a etapa de aceitação regulatória compreende cerca de 6 anos. Algumas diferenças são observadas entre o processo europeu e o norteamericano, os quais estão representados nas Figuras $1 \mathrm{e}$ 2 , respectivamente.

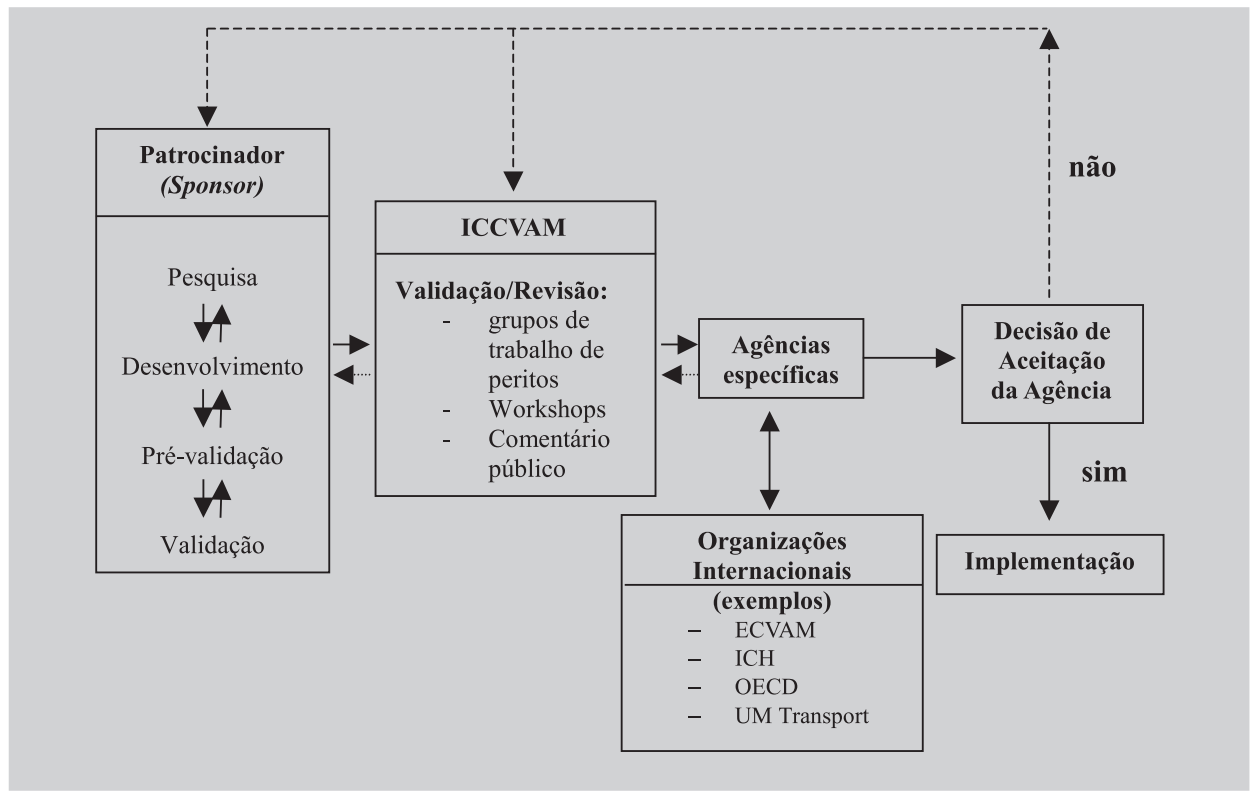

FIGURA 1 - Processo de implementação regulatória nos Estados Unidos.

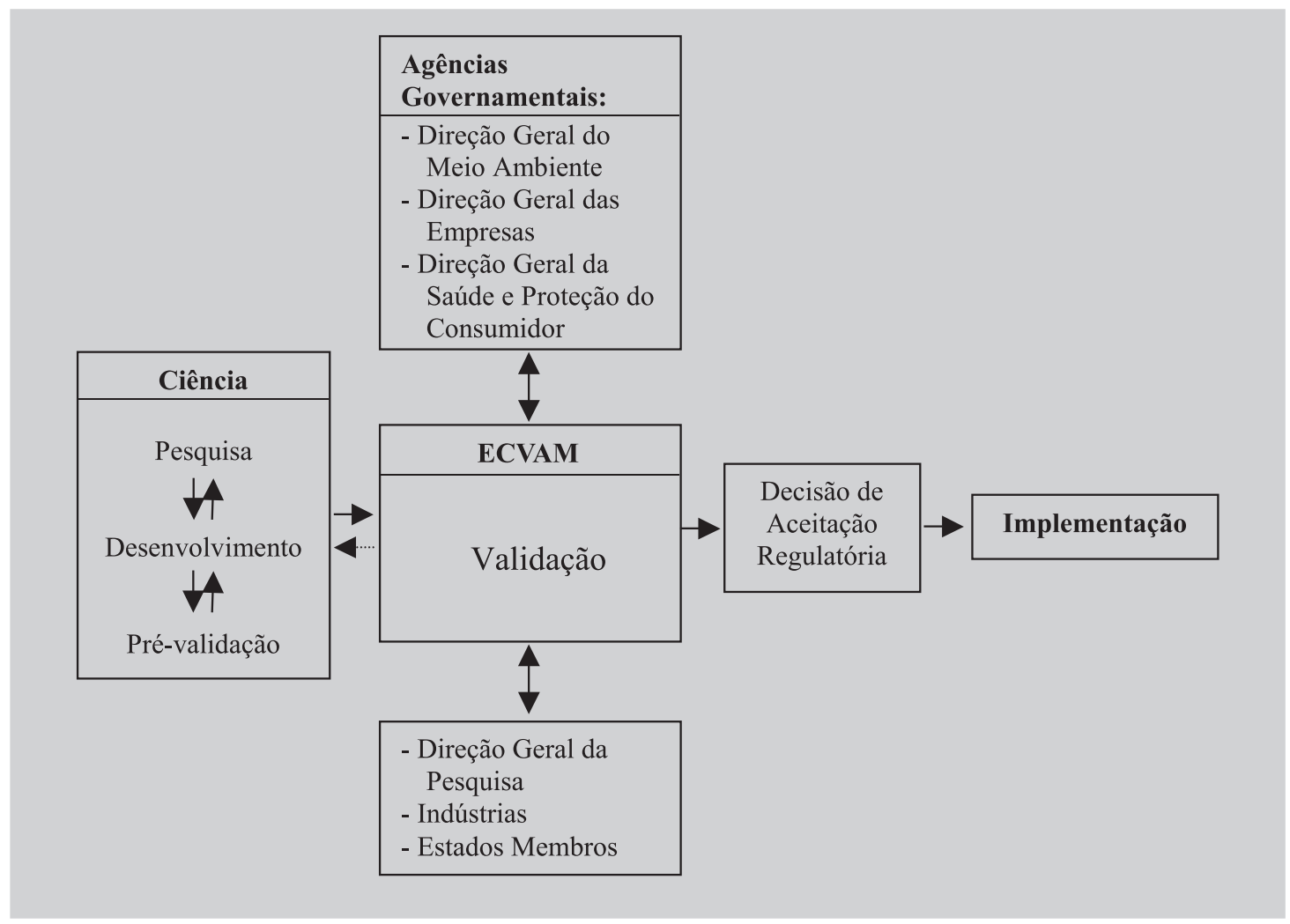

FIGURA 2 - Processo de implementação regulatória na União Européia. 
Ressalta-se que durante todo o processo (desenvolvimento, pré-validação, validação, revisão, aceitação regulatória e implementação) deve ser mantido envolvimento consistente e comunicação apropriada entre todos os interessados (pesquisadores, usuários, reguladores e público), a fim de facilitar a validação e a aceitação dos novos métodos propostos. Da mesma forma, a aceitação regulatória de um novo método deve ser amplamente divulgada aos cientistas e às diversas organizações nacionais e internacionais por intermédio de artigos publicados em jornais e revistas, realização de workshops e outros meios (Olden..., 2004; Stokes, 2002).

A comunicação atualizada e correta dos acontecimentos é um passo de extrema importância para tornar válida as determinações adotadas e para facilitar a harmonização dos novos métodos desenvolvidos, sendo que esta também é impulsionada pela publicação de protocolos por organizações internacionais, tais como a OECD, sendo indispensável a interação entre as Agências de diferentes nacionalidades, sempre com o objetivo de encorajar o processo de harmonização internacional (Richmond, 2002; Olden..., 2004).

\section{DISCUSSÃO E PERSPECTIVAS FUTURAS}

Observa-se imenso avanço, científico, tecnológico e ético quanto ao tema e inclusive intensa discussão em vista das futuras mudanças na condução da avaliação da toxicidade de determinada substância. Assim, a utilização de diversos outros parâmetros deve ser considerada neste processo como ferramenta para avaliação preliminar, tais como, propriedades físico-químicas, relação estrutura-atividade, utilização de modelos computadorizados e condução de métodos in vitro e ex vivo. Neste contexto, a avaliação por etapas (tier approach) tem-se mostrado como tendência de extrema relevância frente aos novos avanços e ao anseio global de redução do uso de animais, visto que muitos dos experimentos in vivo ainda são insubstituíveis.

Atualmente, já existe consenso de que os estudos com animais devem ser conduzidos somente quando: 1) o objetivo é de importância justificável; 2) não existem métodos alternativos válidos; 3 ) todas as estratégias relevantes de redução e refinamento já foram identificadas e implementadas; 4) o desenho e a condução do estudo minimizem o prejuízo causado ao bem estar animal, não somente com relação ao número de animais utilizados, mas também em relação à dor e ao sofrimento causado e 5) exista benefício científico máximo (Richmond, 2002).

Com base neste conceito e na tendência de harmonização internacional, a validação e a implemen- tação de métodos alternativos necessitam do envolvimento de diversas agências, assim como das áreas científica, econômica e política, como ocorre nos Estados Unidos e na Comunidade Européia, por se tratar de processo extremamente complexo, visando à posterior aceitação internacional do método validado.

Diversas modificações nos setores científico e regulatório estão previstas, como já ocorreu com o processo de classificação toxicológica, que resultou na elaboração do Global Harmonization System of Classification and Labelling of Chemicals (GHS), que é um sistema harmonizado de classificação do perigo de substâncias e de misturas de acordo com os efeitos para a saúde e para o ambiente, além de apresentar elementos harmonizados para comunicação do perigo, que devem estar presentes nos rótulos e na ficha de informações de segurança de produtos químicos (FISPQ) ou Material Safety Data Sheet (MSDS).

Entretanto, a discussão sobre a utilização de animais na pesquisa, a intenção de redução do seu uso e o desenvolvimento de novas metodologias ainda têm sido timidamente introduzidos na realidade brasileira. Por esta razão, torna-se de importância significativa a maior discussão e a participação por parte da Academia, da Indústria e dos Órgãos Regulamentadores, visto que estas alterações mundiais afetarão diretamente todos aqueles envolvidos com a pesquisa, o registro e a aprovação de novas substâncias.

\section{ABSTRACT}

\section{Reduction, refinement and replacement of animal use in toxicity testing: an overview}

Toxicity assessment is the process of predicting the adverse effects that may be caused to an organism by exposuring it to a given chemical and, for regulation purposes, the most used model in toxicity testing is the animal. However, in today's society the use of animals has become a subject of much public health due to the large number of animals used as well as the pain and distress caused, mainly related to acute toxicity testing. The concept of the "Three Rs" - reduction, refinement, and replacement of animal use - emerged as a mean of removing inhumanity from animal experimentation and aiming to lower the number of animals used, in order to decrease the incidence or severity of inhumane procedures and to find alternative procedures to replace in vivo testing. Many alternative toxicological tests have already been implemented, even knowing that this process is very complex and need to be extensively validated and accepted by regulatory 
bodies. This paper presents an overview on several aspects of the "Three Rs", including historical evolution and implementation and validation of alternative methods, emphasizing its application on toxicity testing and in global harmonization.

UNITERMS: Three Rs Program. Alternative methods. Experimental animals. Toxicity testing

\section{AGRADECIMENTO}

À Mariana Penteado Soares, pelo auxílio fornecido na normalização das referências.

\section{REFERÊNCIAS BIBLIOGRÁFICAS}

ALTERNATIVE Chronology. in: overview of the issues. [S.I.; s.n.], [entre 1997 e 2003]. Disponível em: <http:// www.hsus.org/ace/ 12443>. Acesso em: 11 jul. 2003.

BALLS, M. Replacement of animal procedures: alternatives in research, education and testing. Lab. Animals, v. 28, p. 193-211, 1994.

BALLS, M.; VAN ZELLER, A.-M; HALDER, M.,eds. Progress in the reduction, refinement and replacement of animal experimentation. Amsterdam: Elsevier, 2000. $1795 \mathrm{p}$.

BARROS, S. B. M; DAVINO, S. C. Avaliação da toxicidade. In: OGA, S. Fundamentos de toxicologia. 2.ed. São Paulo: Atheneu, 2003. cap. 1.5. p. 57-68.

BOTHAM, C.; HOLMES, P.; LEVY, L. S.; HARRISON, P. T. C. Prospects for the use of alternative test methods. Leicester: MRC Institute for Environment and Health, 2001. Disponível em: <http:/www.le.ac.uk/ieh/ pdf/HoLfinal.pdf $>$. Acesso em: 10 maio 2003.

BOTHAM, P. A. Acute systemic toxicity. ILAR J., v. 43, supl., p. S27-S30, 2002.

CORRÊA, C. L.; ALONZO, H. G. A.; TREVISAN, R.M.S. Avaliação do risco. In: OGA, S. Fundamentos de toxicologia. 2. ed. São Paulo: Atheneu, 2003. cap. 1.6. p. 69-76.

DIPASQUALE, L. C.; HAYES, A. W. Acute toxicity and eye irritancy. In: HAYES, A. W. Principles and methods of toxicology. 4.ed. London: Taylor \& Francis, 2001. cap. 18, p. 853-916.
ECOBICHON, D. J., ed. The basis of toxicity testing. 2. ed. Boca Raton: CRC Press, 1997. 220 p.

EUROPEAN CENTRE FOR THE VALIDATION OF ALTERNATIVE METHODS. The SIS databases provide factual information on alternative methods to animal experiments at any stage of development and validation. [S.1: s. n.], [200-?]. Disponível em: $<\mathrm{http}: / /$ ecvam.jrc.cec.eu.int/index.htm>. Acesso em: 20 jul. 2003.

EXECUTIVE COMMITTEE OF THE CONGRESS. Background to the three Rs declaration of Bologna, as adopted by the $3^{\text {rd }}$ World Congress on Alternatives and Animal Use in the Life Sciences, Bologna, Italy, on 31 August 1999. In: BALLS, M.; VAN ZELLER, A.-M; HALDER, M.,eda. Progress in the reduction, refinement and replacement of animal experimentation. Amsterdam: Elsevier, 2000. p. 17-22.

FESTING, M.F.W. Reduction of animal use: experimental design and quality of experiments. Lab. Animals, v. 28, p. 212-221, 1994.

FLECKNELL, P.A. Refinement of animal use - assessment and alleviation of pain and distress. Lab. Animals, v. 28, p. 222-231, 1994.

HUMANE Society of United States (HSUS). Washington: [s.n.], 2003. Disponível em: <http://www.hsus.org/ace/ 352>. Acesso em: 11 jul. 2003.

INTERAGENCY COORDINATING COMMITTEE ON THE VALIDATION OF ALTERNATIVE METHODS (ICCVAM). Annual Progress Report. [S.1.]: National Institute of Health, 2001. Disponível em: <http:// iccvam.niehs.nih.gov/about/annrpt/annrpt01.pdf $>$. Acesso em: 07 jul. 2003.

INTERAGENCY COORDINATING COMMITTEE ON THE VALIDATION OF ALTERNATIVE METHODS (ICCVAM). Evaluation of the validation status of toxicological methods: General guidelines for submissions to ICCVAM. [S.1.]: National Institute of Health, rev. out, 1999. NIH Publication No. 99-4496. Disponível em: <http://iccvam.niehs.nih.gov/docs/ guidelines/subguide.htm>. Acesso em: 05 jun. 2003. 
INTERAGENCY COORDINATING COMMITTEE ON THE VALIDATION OF ALTERNATIVE METHODS (ICCVAM). The national toxicology program interagency center for the evaluation of alternative toxicological methods (NICEATM). [S.1.: s.n.], 2003. Disponível em: $<\mathrm{http}: / /$ iccvam.niehs.nih.gov $>$. Acesso em: 07 jul. 2003.

INTERAGENCY COORDINATING COMMITTEE ON THE VALIDATION OF ALTERNATIVE METHODS (ICCVAM). Validation and regulatory acceptance of toxicological test methods: A Report of the ad hoc interagency coordinating committee on the validation of alternative methods. [S.1.]: National Institute of Health, 1997. Publication NIH No: 97-3981. Disponível em: $<$ http://iccvam.niehs.nih.gov/docs/guidelines/ validate.pdf $>$. Acesso em: 04 ago. 2003.

KOETER, H. B. W. M. Development, implementation and use of alternative test methods in regulatory hazard assessment in OECD member countries. Environment, health and safety division, OECD, [2003?]. Formato PDF. 1 apresentação, Power Point. Disponível em: $<$ http:// europa.eu.int/comm/research/info/conferences/rrr/ppt/ koeter.pdf $>$. Acesso em: 23 ago. 2003.

KOETER, H. B. Test guideline development and animal welfare: regulatory acceptance of in vitro studies. Reprod. Toxicol., v. 7, supl. 1, p. 117-123, 1993.

MATFIELD, M. (Dir). Research Defence Society (RDS): Understanding animal research in medicine. London: [s.n.], 2003. Disponível em: <http://www.rdsonline.org.uk>. Acesso em: 21 jun. 2003.

MEYER, O. Testing and assessment strategies, including alternative and new approaches. Toxicol. Letters, v. 140141, p. 21-30, 2003.

OLDEN, K. (Dir). National Toxicology Program. [S.l.: s.n.], 2004. Disponível em: $<$ http://ntp-server.niehs.nih.gov/>. Acesso em: 20 jan. 2004.

ORGANIZATION FOR ECONOMIC CO-OPERATION AND DEVELOPMENT (OECD). OECD Guideline for the testing of chemicals: acute oral toxicity - Up-anddown procedure. Paris: OECD, 2000. Guideline 425. Disponível em: $<$ http://www.oecd.org/home/>. Acesso em: 01 out. 2001
ORGANIZATION FOR ECONOMIC CO-OPERATION AND DEVELOPMENT (OECD). OECD Guideline for the testing of chemicals: Acute oral toxicity. Paris: OECD, 1987. Guideline 401. Disponível em: <http:// www.oecd.org/home/>. Acesso em: 01 out. 2001.

ORGANIZATION FOR ECONOMIC CO-OPERATION AND DEVELOPMENT (OECD). Harmonized integrated classification system for human health and environmental hazards of chemical substances and mixtures. Paris: OECD, 2001. Disponível em: <http:// www.oecd.org/ehs/class/HCL6.htm>. Acesso em: 13 dez. 2001

ORGANIZATION FOR ECONOMIC CO-OPERATION AND DEVELOPMENT (OECD). OECD Guideline for the testing of chemicals: Acute oral toxicity - Acute toxic class method. Paris: OECD, 1996. Guideline n. 423. Disponível em: $<$ http://www.oecd.org/home/>. Acesso em: 11 nov. 2003.

ORGANIZATION FOR ECONOMIC CO-OPERATION AND DEVELOPMENT (OECD). OECD Guideline for the testing of chemicals: Acute oral toxicity - Fixed dose method. Paris: OECD, 1992. Guideline n. 420. Disponível em: <http://www.oecd.org/home/>. Acesso em: 11 nov. 2003.

ORGANIZATION FOR ECONOMIC CO-OPERATION AND DEVELOPMENT (OECD). OECD Guideline for the testing of chemicals: In vitro $3 \mathrm{~T} 3 \mathrm{NRU}$ photoxicity test. Paris: OECD, 2002a. Guideline Draft TG 432. Disponível em: <http://www.oecd.org/home/>. Acesso em: 11 nov. 2003.

ORGANIZATION FOR ECONOMIC CO-OPERATION AND DEVELOPMENT (OECD). OECD Guideline for the testing of chemicals: In vitro skin corrosion: Human skin model test. Paris: OECD, 2002b. Guideline Draft TG 431. Disponível em: <http://www.oecd.org/ home/>. Acesso em: 11 nov. 2003.

ORGANIZATION FOR ECONOMIC CO-OPERATION AND DEVELOPMENT (OECD). OECD Guideline for the testing of chemicals: In vitro skin corrosion: Transcutaneous eletrical resistance test (TER). Paris: OECD, 2002c. Guideline Draft TG 430. Disponível em: $<$ http://www.oecd.org/home/>.Acesso em: 11 nov. 2003. 
RICHMOND, J. Refinement, reduction, and replacement of animal use for regulatory testing: future improvements and implementation within the regulatory framework. ILAR Journal, v. 43, supl., p. S63-S68, 2002.

RUSSEL, W. M. S.; BURCH, R. L. The principles of humane experimental technique. London: Universities Federation for Animal Welfare (UFAW), 1992. ISBN: 0900767782. Special Edition. Disponível em: $<$ http:// altweb.jhsph.edu/publications/humane_exp/het-toc.htm>. Acesso em: 14 maio 2003.

SCHECHTMAN, L. M. Implementation of the $3 \mathrm{Rs}$ (Refinement, reduction, and replacement): Validation and regulatory acceptance considerations for alternative toxicological test methods. ILAR Journal, v. 43, supl., p. S85-S94, 2002.

SCHLEDE, E.; DIENER, W.; GERNER, I. Humane endpoints in toxicity testing. In: HUMANE endpoints in animals experiments for biomedical research. London: Royal Society of Medicine Press, [entre 1998 e 2003]. p. 75-78. Disponível em: <www.lal.org.uk/pdffiles/ schlede.pdf $>$. Acesso em: 14 jul. 2003.

SPIELMANN, H. Animal use in the safety evaluation of chemicals: harmonization and emerging needs. ILAR Journal, v. 43, supl., p. S11- S17, 2002.

STEPHENS, M. L., GOLDBERG, A. M., ROWAN, A. N. The first forty years of the alternatives approach: refining, reducing, and repalcing the use of laboratory animals. In: ROWAN, A. N. The state of the animals 2001. Washington: Humane Society Press, 2001. chapter 8. Disponível em: $<$ http://files.hsus.org/web-files/PDF/ MARK_State_of_Animals_Ch_08.pdf $>$.Acesso em: 15 fev. 2003.
STITZEL, K.A. Tiered testing strategies - Acute local toxicity. ILAR J., v. 43, supl., p. S21-S26, 2001.

STOKES, W. S. Humane endpoint for laboratory animals used in regulatory testing. ILAR J., v. 43, supl., p. S31-S38, 2002.

THREE Rs Declaration of Bologna. In: BALLS, M.; VAN ZELLER, A.-M; HALDER, M., eds. Progress in the reduction, refinement and replacement of animal experimentation. Amsterdam: Elsevier, 2000. p. 15.

WHITE, W. J. The use of laboratory animals in toxicologic research. In: HAYES, A. W. Principles and methods of toxicology. 4.ed. London: Taylor \& Francis, 2001. cap. 16, p. 773-818.

WORTH, A. P.; BALLS, M. The principles of validation and the ECVAM validation process. Altern. Lab. Anim., v. 30, supl. 2, p. 15-21, 2002.

ZURLO, J.; RUDACILLE, D.; GOLDBERG, A. M. Animal and alternatives in testing: History, science, and ethics. Larchmont: The Johns Hopkins University, 2002. Disponível em: $<$ http://caat.jhsph.edu/pubs/animal_alts/ animal_alts.htm>. Acesso em: 14 jul. 2003.

Recebido para publicação em 08 de março de 2004. Aceito para publicação em 06 de agosto de 2004. 